\title{
Evaluation of Bread Wheat (Triticum Aestivum L.) Genotypes for Stem and Yellow Rust Resistance in Ethiopia
}

\author{
Wondwesen Shiferaw ${ }^{1, ~ *, ~ M o h a m m e d ~ A b i n a s a ~}{ }^{1}$, Wuletaw Tadesse ${ }^{2}$ \\ ${ }^{1}$ International Center for Agricultural Research in the Dry Areas (ICARDA), Addis Ababa, Ethiopia \\ ${ }^{2}$ International Center for Agricultural Research in the Dry Areas (ICARDA), Rabat, Morocco
}

Email address:

wondehildana@gmail.com (W. Shiferaw)

${ }^{*}$ Corresponding author

\section{To cite this article:}

Wondwesen Shiferaw, Mohammed Abinasa, Wuletaw Tadesse. Evaluation of Bread Wheat (Triticum Aestivum L.) Genotypes for Stem and Yellow Rust Resistance in Ethiopia. Computational Biology and Bioinformatics. Vol. 8, No. 2, 2020, pp. 43-51.

doi: $10.11648 /$ j.cbb.20200802.13

Received: September 21, 2020; Accepted: October 5, 2020; Published: October 30, 2020

\begin{abstract}
Wheat production in Ethiopia is challenged by different biotic stress. Among these biotic stresses, stem rust (Puccinia graminis f. sp. Tritici) and yellow rust (P. striiformis Westend. f. sp. Tritici) are the most devastating.. Improvement of wheat genotypes through incorporation of resistant genes to stem rust and yellow rust and testing them under hot spot areas is the most economical and environmentally friendly approach to develop resistant cultivars. Field experiment using an augmented design was undertaken at Kulumsa during 2016/17 and 2017/18 cropping season to evaluate the response of 119 elite spring bread wheat genotypes and three checks for stem and yellow rust. Based on the disease severity $71.4 \%$ and $96.6 \%$ of the genotypes showed the lowest score $(0-10 \%)$ for stem rust in the first and second cropping season, respectively. About $59.7 \%$ and $66.4 \%$ of the genotypes were also showed the lowest disease severity $(0-10 \%)$ for yellow rust during $2016 / 17$ and $2017 / 18$ cropping season, respectively. The genotypes showed significant $(\leq 0.05)$ difference in Area Under Disease Progress Curve (AUDPC) for stem rust and yellow rust during 2016/17 and 2017/18 cropping season but there was significant difference $(\leq 0.05)$ in Coefficient of Infection $(\mathrm{CI})$ for stem rust during the first cropping season only. The genotypes exhibited significant difference $(\leq 0.01)$ and $(\leq 0.001)$ in $\mathrm{CI}$ for yellow rust in the first and second cropping season, respectively. Negative association of grain yield and thousand kernel weight with stem and yellow rust was found in both cropping season. Among the genotypes ASEEL-1//MILAN/PASTOR/3/SHAMISS-3, ZERBA6/FLAG6/3/TAM200/PASTOR//TOBA97, ZERBA6/FLAG6/3/TAM200/PASTOR//TOBA97, NJOROSD-2/SHIHAB-12 and ICBW 206971//SHUHA-4/CHAM8/3/SIRAJ are highly resistant for both yellow and stem rust in both cropping season.
\end{abstract}

Keywords: Bread Wheat, Stem Rust, Yellow Rust, Resistance, Susceptible

\section{Introduction}

Bread wheat (TriticumaestivumL.) plays a significant role to food security in the world. It provides more than $21 \%$ of the calories and $20 \%$ of the protein and its demand in developing world becomes increasing from time to time [10]. Bread wheat is the main cereal crop in Ethiopia, cultivated on about 1.7 million ha with estimated annual production of 4.5 million tons [5]. Ethiopia is the second largest wheat producer in sub Saharan Africa, and wheat is becoming a major crop for improving food security in this country [8,
12]. However, its production is challenged by different biotic stress such as wheat rusts [2, 37].

Stem rust caused by Puccinia graminis f. $s p$. Tritici is one of the fungal diseases which cause severe yield loss in wheat [14]. It is aggravated under warm and moist environmental conditions [18]. Stem rust was detected in Ethiopia in the early 1993 [17]. According to [22] about 40000 ha were infected with wheat stem rust in 2013/2014 cropping season in this country. The stem rust epidemics frequently occurs in the highlands of Ethiopia such as Oromiya, Southern Nation Nationality Peoples, Tigray, and Amhara regions [25] due to 
the bimodal rainfall patterns which facilitates transferring of inoculums from one season to the next $[24,25]$ and it may causes $100 \%$ yield losses and reduction of grain quality $[7,9]$ under sever condition.

Yellow rust caused by $P$. striiformis Westend. f. sp. tritici (Pst) is the most important wheat rust disease which causes $100 \%$ yield losses [43] in susceptible varieties. Although, temperate regions with cool and wet weather conditions are suitable for the development of this pathogen [44] since 2000 , destructive races of yellow rust adapted to higher temperature [27] have been observed across the world. In Ethiopia yellow rust epidemics has been reported since 1970 's [35]. It causes yield loss of 70-100\% in susceptible cultivars like Kubsa and Dashen [36] and as well as yield and quality loss in other cultivars [30, 31, 33]. A devastating yellow rust epidemic in Ethiopia affected more than 600,000 ha of wheat in 2010 cropping season [29]. Therefore, it is an economically important disease of wheat in the country [28, 31].

Use of resistant cultivars and chemicals are the major options to control rusts $[11,16]$. However, controlling these rust using chemicals is not affordable by resource poor farmers because of high cost and lack of timely supply [1]. Furthermore, chemicals are not environmentally friendly. Genetic resistance is the most suitable approach to control rust diseases in wheat [6]. Although a number of bread wheat cultivars have been released from germplasms introduced from CIMYT and ICARDA, most of the varieties became susceptible for stem and yellow rust within short periods after release [41]. Thus, continuous evaluation of wheat germplasm in stem and yellow rust hot spot areas like Kulumsa is vital to select for adult plant resistance [14]. Therefore, the present study was carried out to evaluate elite bread wheat genotypes from ICARDA in order to identify sources of resistance for stem and yellow rust.

\section{Materials and Methods}

A field experiment was conducted at Kulumsa Agricultural Research Center of Ethiopian Agricultural Research Institute (EIAR). Kulumsa is located at $8^{\circ} 00^{\prime} \mathrm{N}$ and $39^{\circ} 07^{\prime} \mathrm{E}$, and $2210 \mathrm{~m}$ above sea level in Arsi Administrative Zone of Oromiya Regional State, $167 \mathrm{~km}$ South East of Addis Ababa. The agro-climatic condition of the area is wet and receives a unimodal mean annual rainfall of $809.15 \mathrm{~mm}$ from March to September; however, the peak season is from July to August. The maximum and minimum mean temperature is 23.1 and $9.9^{\circ} \mathrm{C}$, respectively [19].

One hundred nineteen Elite Spring Bread Wheat genotypes from the International Center for Agricultural Research Center in the Dry Areas (ICARDA) and 3checks (Hidasse, Kingbird and Shorima) obtained from the Kulumsa Agricultural Research Center were used for this study. The checks were used to compare the resistance of these genotypes to stem and yellow rust. The mixture of three bread wheat cultivar (Digelu, Kubssa, Morocco) susceptible for stem and yellow rust were used as spreader and planted in both sides of each block to ensure production of sufficient inoculums to provide uniform infection.

The genotypes were planted using an augmented design in a plot size of $3.0 \mathrm{~m}$ length, planted in six rows with $0.2 \mathrm{~m}$ spacing between rows at Kulumsa during cropping season of 2016/201 and 2017/2018. Field managements and agronomic practices were conducted as recommended.

Days to $50 \%$ heading and days to $90 \%$ physiological maturity were taken. At physiological maturity five random plants within each plot were used to determine plant height. Grain yield and 1000 kernel weight were measured.

The modified Cobb's scale [20] was used to assess the disease severity. The reaction response of the genotype's to the infection was scored three times at 12 days interval starting from the mid of September when diseases symptom started. There action types were designated by " $R$ " or resistant (small uredinia surrounded by chlorosis or necrosis); "MR" or moderately resistant (medium size uredinia surrounded by chlorosis or necrosis); "MS" or moderately susceptible (medium large compatible uredinia without chlorosis and necrosis); and "S" or susceptible (large, compatible uredinia without chlorosis and necrosis). The disease severity was scored in the percentage of 0 to 100 scale [14].

The disease severity data and host reaction response were combined to calculate the coefficient of infection (CI) following [21] by multiplying severity value with constant values of $0,0.2,0.4,0.6,0.8$, or 1 for host response ratings of immune (I), resistant (R), moderately resistant (MR), intermediate (M), moderately susceptible (MS), or susceptible (S), respectively. Genotypes with coefficient of infections ranging 0 to 20,20 to 30,30 to 40,40 to 60 and 60 to 100 were resistant, moderately resistant, moderately susceptible, moderately susceptible to susceptible and susceptible, respectively. Susceptibility and resistance comparison of the studied genotypes' was done by calculating Area Under Disease Progress Curve (AUDPC) according to the method of [8] as: $A U D P C=\sum i=1 n-1 \quad[(t i+1-t i)(y i+y i+1) / 2]$. Where " $t$ " is time in days of each reading, " $y$ " is the percentage of affected foliage at each reading and " $n$ " is the number of readings. Diseases severity score and the coefficient of infection were used to compute AUDPC.

Analysis of variance was conducted on different diseases parameters such as AUDPC, CI, and disease severity to determine resistance differences among the studied elite bread wheat genotypes. The data were analyzed using $\mathrm{R}$ software [39]. A correlation coefficient was computed to estimate the association between diseases and agronomic traits including yield and yield components.

\section{Results}

\subsection{Responses to Stem and Yellow Rusts}

Significant difference $(\leq 0.05)$ was observed among the tested genotypes for yellow rust during 2017/18 cropping season (Table 1). In contrast the genotypes didn't show 
significant difference for stem rust in both cropping season. AUDPC and CI for stem rust showed significant variation $(\leq 0.05)$ among the studied genotypes in the first cropping season (Table 1). The genotypes showed significant difference $(\leq 0.01)$ and $(\leq 0.001)$ in CI for yellow rust in the first and second cropping season, respectively (Tables 1 and $2)$. The Genotypes also exhibited significant variation $(\leq 0.01)$ in AUDPC for yellow rust in the second cropping season (Table 2). The Checks showed significant variation $(\leq 0.01)$ only in AUDPC during 2016/17 season for stem rust (Table 1) in contrast there was no significant difference among the checks in CI for stem and yellow rust in both seasons.

The frequency distributions of the yellow rust severity scores for elite bread wheat genotypes for both cropping season are presented in figure 1. About Eighty and Seventy elite genotypes exhibited the lowest scores (0-10\%) in the first and second cropping season, respectively (Figure 1). The highest scores $(41-50 \%)$ for yellow rust were observed for only four genotypes in the second cropping season (Figure $1)$.

Genotypes, ASEEL-1//MILAN/PASTOR/3/SHAMISS-3, ZERBA6/FLAG6/3/TAM200/PASTOR//TOBA97, ZERBA6/FLAG6/3/TAM200/PASTOR//TOBA97 were among the highly resistant genotypes for both yellow rust and stem rust with severity levels of 0,5 , and 5 , respectively.

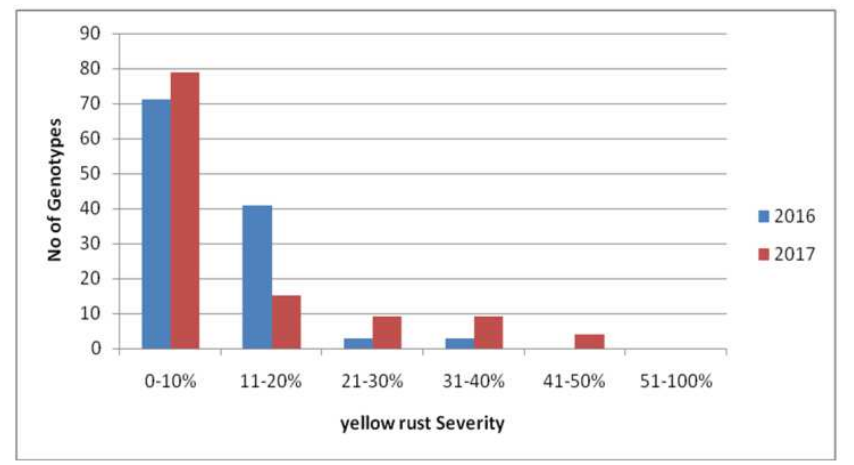

Figure 1. Histogram of yellow rust severity during 2016 and 2017 cropping season.

\subsection{Variability for Agronomic Traits}

The genotypes showed highly significant difference $(\leq 0.001)$ for days to $50 \%$ heading and days to $90 \%$ physiological maturity (Table 1) in the first cropping season but in the second cropping season they showed significance variation $(\leq 0.01)$ and $(\leq 0.05)$ for days to $50 \%$ heading and days to $90 \%$ physiological maturity, respectively (Table 2 ). There was no significant variation among the checks for these traits in both seasons. The studied genotypes also showed significant variation $(\leq 0.05)$ for plant height during 2016/17 (Table 1).

Significant difference $(\leq 0.05)$ was observed among the genotypes for thousand kernel weight in both cropping seasons (Tables 1 and 2) but there was no significant difference among the genotypes for grain yield during both cropping seasons.

\subsection{Association Among Traits}

The Pearson's correlation coefficient analysis showed that days to $50 \%$ flowering, days to maturity, plant height, thousand kernels weight and grain yield were negatively correlated with yellow and stem rust in the first cropping season (Table 3 ). In the second cropping season grain yield and thousand kernel weight were negatively associated with stem and yellow rust (Table 3 ).

About eighty five and one hundred fifteen genotypes out of the studied genotypes showed the lowest score $(0-10 \%)$ for stem rust in 2016/17 and 2017/18 cropping season, respectively (Figure 2). In both cropping season the genotypes did not show more than $30 \%$ severity level for stem rust (Figure 2).

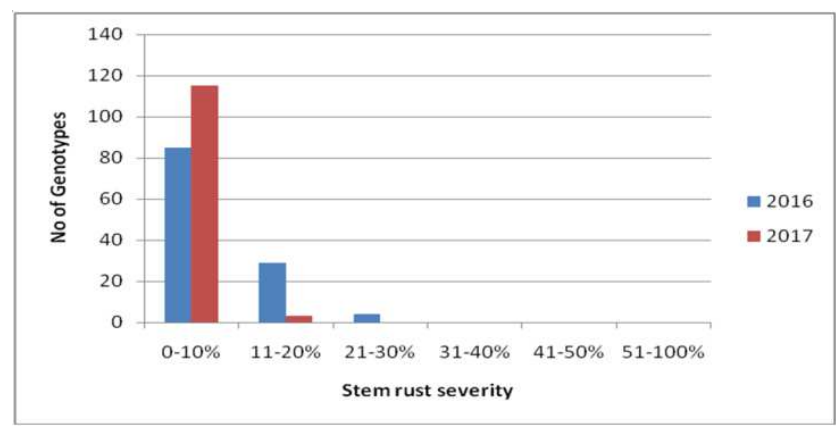

Figure2. Histog ram of stem rust severity during 2016 and 2017 cropping season.

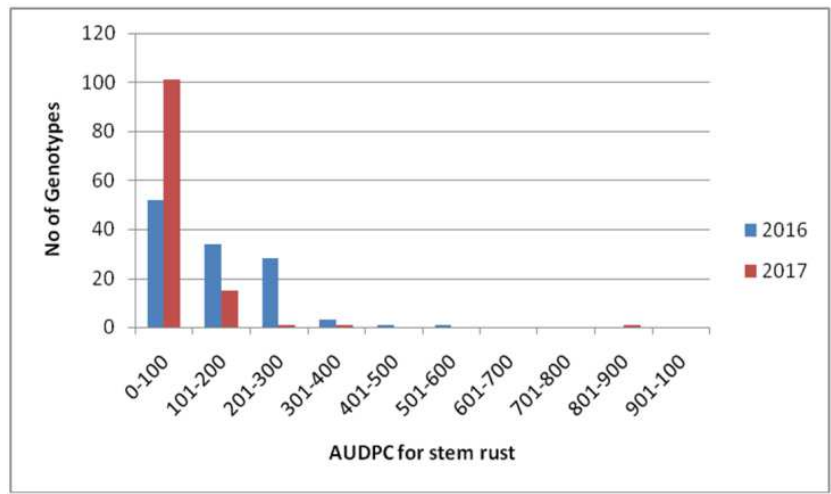

Figure 3. Histogram of Area Under Disease progress Curve for stem rust during 2016 and 2017 cropping season.

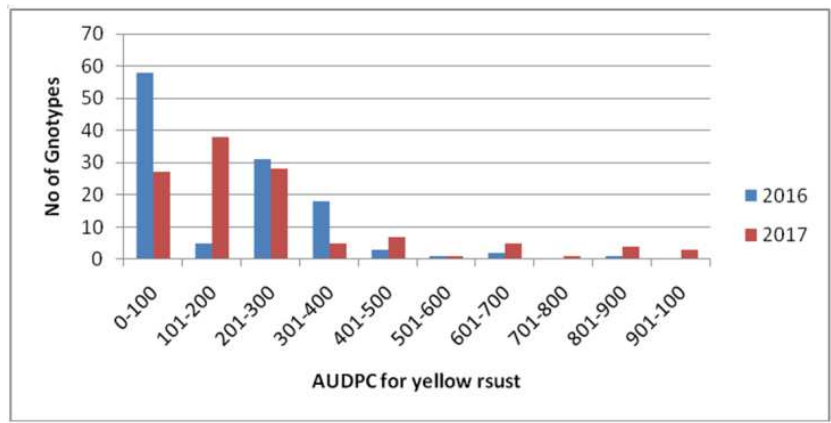

Figure 4. Histogram of Area Under Disease progress Curve for yellow rust during 2016 and 2017 cropping season. 
Table 1. Analysis of variance for the various traits of the genotypes during 2016/17 cropping season at Kulumssa.

\begin{tabular}{lllllllllllll}
\hline \multicolumn{10}{c}{ Stem Rust } & \multicolumn{1}{l}{ Yellow Rust } \\
\hline Source of Variation & Df & CI & AUDPC & CI & AUDPC & DH & DM & PH (cm) & TKW (g) & $\begin{array}{l}\text { GY } \\
(\text { Kg/plot) }\end{array}$ & YR & SR \\
\hline Block (Adj) & 3 & $2.2^{\text {ns }}$ & $4200^{\text {ns }}$ & $8.4^{\text {ns }}$ & $26475^{\text {ns }}$ & $137.2^{\text {ns }}$ & $6.1^{\text {ns }}$ & $0.3^{\text {ns }}$ & $1.61^{\text {ns }}$ & $0.13^{\text {ns }}$ & $81.0^{\text {ns }}$ & $33.3^{\text {ns }}$ \\
Treat (Adj) & 121 & $30.7^{*}$ & $10405^{*}$ & $21.2^{* *}$ & 30639 & $34.4^{* * *}$ & $38.3^{* * *}$ & $96.2^{*}$ & $22.12^{*}$ & $1.363^{*}$ & $14.6^{\text {ns }}$ & $44.8^{\text {ns }}$ \\
Controls & 2 & $100.3^{\text {ns }}$ & $32025^{* *}$ & $0.3^{\text {ns }}$ & $8400^{\text {ns }}$ & $32025^{\text {ns }}$ & $4.1^{\text {ns }}$ & $120.6^{*}$ & $42.62^{*}$ & $6.9^{* *}$ & $14.6^{\text {ns }}$ & $214.6^{* *}$ \\
AugmentedVsControls & 1 & $5.3^{\text {ns }}$ & $4811^{\text {ns }}$ & $27.6^{*}$ & 66327 & $18.9^{*}$ & $126.1^{\text {ns }}$ & $0.52^{\text {ns }}$ & $8.19^{* *}$ & $174.9^{\text {ns }}$ & $1.5^{\text {ns }}$ & $0.4^{\text {ns }}$ \\
Augmented & 118 & $36.7^{*}$ & $11836^{*}$ & $22.4^{* *}$ & $32552^{\text {ns }}$ & $38.6^{* * *}$ & $41.6^{* * *}$ & $106.6^{*}$ & $22.27^{*}$ & $1.29^{\text {ns }}$ & $84.3^{\text {ns }}$ & $50.4^{\text {ns }}$ \\
Residual & 6 & 6.1 & 2025 & 2.1 & 12000 & 1.9 & 1.4 & 22.8 & 5.1 & 0.36 & 18.7 & 14.6 \\
\hline
\end{tabular}

Wherens, *****and*, non significant, Significantly different at $0.001,0.01$ and 0.05 , respectively. Df=Degree of freedom, CI $=$ Coefficient of infection, $\mathrm{AUDPC}=$ Area under disease progress curve, $\mathrm{DH}=$ Days to $50 \%$ heading, $\mathrm{DM}=$ Days to maturity, $\mathrm{PH}=\mathrm{Plant}$ Height, $\mathrm{TKW}=\mathrm{Thousand}$ kernel weight, $\mathrm{GY}=$ grain yield, $\mathrm{YR}=$ yellow rust and $\mathrm{SR}=$ stem rust.

Table2. Analysis of variance for the various traits of the genotypes during 2017/18 cropping season at Kulumssa.

\begin{tabular}{|c|c|c|c|c|c|c|c|c|c|c|c|c|}
\hline \multirow[b]{2}{*}{ Source of Variation } & \multirow[b]{2}{*}{ Df } & \multicolumn{2}{|c|}{ Stem Rust } & \multicolumn{9}{|c|}{ Yellow Rust } \\
\hline & & CI & AUDPC & CI & AUDPC & DH & DM & PH (cm) & TKW (g) & $\begin{array}{l}\text { GY } \\
\text { (Kg/plot) }\end{array}$ & YR & SR \\
\hline Block (Adj) & 3 & $25.6^{\mathrm{ns}}$ & $7300^{\text {ns }}$ & $4^{\mathrm{ns}}$ & $9875^{\mathrm{ns}}$ & $4.6^{\mathrm{ns}}$ & $6.9^{\text {ns }}$ & $3.8^{\mathrm{ns}}$ & $5.06^{\mathrm{ns}}$ & $0.014^{\mathrm{ns}}$ & $25.0^{\mathrm{ns}}$ & $27.8^{\mathrm{ns}}$ \\
\hline Treat (Adj) & 121 & $20.7^{\text {ns }}$ & $8316^{\mathrm{ns}}$ & $72.3 * * *$ & $46515^{*}$ & $44.7 *$ & $39.6^{*}$ & $75.7^{*}$ & $23.15^{*}$ & $0.374^{\mathrm{ns}}$ & $116.2 *$ & $31.2^{\mathrm{ns}}$ \\
\hline Controls & 2 & $22.3^{\text {ns }}$ & $7275^{\text {ns }}$ & $0.3^{\mathrm{ns}}$ & $5925^{\mathrm{ns}}$ & $1.7^{\mathrm{ns}}$ & $31^{\mathrm{ns}}$ & $248.1 * *$ & $85.69 * *$ & $7.21 * *$ & $2.1^{\mathrm{ns}}$ & $27.1^{\mathrm{ns}}$ \\
\hline Augmented Controls & 1 & $37.0^{\mathrm{ns}}$ & $28596^{\mathrm{ns}}$ & $28.3 *$ & $944^{\mathrm{ns}}$ & $10.3^{\text {ns }}$ & $0.8^{\text {ns }}$ & $279.8 * * *$ & $8.19 * *$ & $11.713 * *$ & $0.4^{\mathrm{ns}}$ & $78.0^{\mathrm{ns}}$ \\
\hline Augmented & 118 & $20.3^{\text {ns }}$ & $8154^{\mathrm{ns}}$ & $76.5 * * *$ & $49141 * *$ & $49.4 * *$ & $42.8^{*}$ & $70.9^{\text {ns }}$ & $22.34 *$ & $0.159^{\text {ns }}$ & $124.1 *$ & $30.8^{\mathrm{ns}}$ \\
\hline Residual & 6 & 20.6 & 7375 & 3 & 6725 & 7.0 & 9.9 & 19.5 & 3.66 & 0.34 & 31.3 & 29.9 \\
\hline
\end{tabular}

Where ns, $* * * * *$ and $*$, non significant, Significantly different at $0.001,0.01$ and 0.05 , respectively. Df=Degree of freedom, CI $=$ Coefficient of infection, $\mathrm{AUDPC}=$ Area under disease progress curve, $\mathrm{DH}=$ Days to $50 \%$ heading, $\mathrm{DM}=$ Days to maturity, $\mathrm{PH}=\mathrm{Plant}$ Height, $\mathrm{TKW}=\mathrm{Thousand}$ kernel weight, $\mathrm{GY}=$ grain yield, $\mathrm{YR}=$ yellow rust and $\mathrm{SR}=$ stem rust

Table 3. Pearson's correlation coefficients among the different diseases and agronomic traits during 2016/17 (Above diagonal) and 2017/18 (Below diagonal) cropping season.

\begin{tabular}{|c|c|c|c|c|c|c|c|c|}
\hline & & YR & SR & DH & DM & PH & TKW & GY \\
\hline YR & 1 & 1 & $-0.01^{\mathrm{ns}}$ & $-0.33^{* *}$ & $-0.19^{*}$ & $-0.01^{\mathrm{ns}}$ & $-0.25^{* *}$ & $-0.07^{\mathrm{ns}}$ \\
\hline SR & $-0.45^{\mathrm{ns}}$ & 1 & 1 & $-0.27^{* *}$ & $-0.19^{*}$ & $-0.13^{\mathrm{ns}}$ & $-0.3^{\text {ns }}$ & $-0.04^{\mathrm{ns}}$ \\
\hline DH & $-0.17^{\text {ns }}$ & $0.13^{\text {ns }}$ & 1 & 1 & $0.79^{* *}$ & $0.37^{* *}$ & $-0.9^{\text {ns }}$ & $0.11^{\mathrm{ns}}$ \\
\hline DM & $-0.14^{\mathrm{ns}}$ & $0.21^{*}$ & $0.76^{* *}$ & 1 & 1 & $0.61^{* *}$ & $-0.9^{\text {ns }}$ & $0.11^{*}$ \\
\hline PH & $0.9^{\mathrm{ns}}$ & $0.51^{\mathrm{ns}}$ & $0.16^{\mathrm{ns}}$ & $0.04^{\mathrm{ns}}$ & 1 & 1 & $0.11^{\mathrm{ns}}$ & $0.24^{* *}$ \\
\hline TKW & $-0.15^{\mathrm{ns}}$ & $-0.02^{\text {ns }}$ & $0.73^{\mathrm{ns}}$ & $0.13^{\mathrm{ns}}$ & $0.05^{\mathrm{ns}}$ & 1 & 1 & $0.09^{\text {ns }}$ \\
\hline GY & $-0.35^{* *}$ & $-0.12^{\mathrm{ns}}$ & $0.48^{\mathrm{ns}}$ & $0.11^{\mathrm{ns}}$ & $-0.01^{\mathrm{ns}}$ & $0.37^{* *}$ & 1 & 1 \\
\hline
\end{tabular}

Where ** and *, Significantly different at 0.01 and 0.05 , respectively. $\mathrm{DH}=$ Days to $50 \%$ heading, $\mathrm{DM}=\mathrm{Days}$ to maturity, $\mathrm{PH}=\mathrm{Plant} \mathrm{Height}$, TKW=Thousand kernel weight, $\mathrm{GY}=$ grain yield, $\mathrm{YR}=$ yellow rust and $\mathrm{SR}=$ stem rust.

Table 4. Stem and yellow rust terminal diseases scores, coefficient of infection (CI) of resistant elite spring bread wheat genotypes planted during 2016/17 and 2017/18 cropping season at Kulumsa.

\begin{tabular}{|c|c|c|c|c|c|c|c|c|}
\hline \multirow{2}{*}{ Pedigree } & \multicolumn{2}{|c|}{2016 (Stem Rust) } & \multicolumn{2}{|c|}{2017 (Stem Rust) } & \multicolumn{2}{|c|}{2016 (Yellow Rust) } & \multicolumn{2}{|c|}{2016 (Yellow Rust) } \\
\hline & Disease score & CI & Disease score & CI & Disease score & CI & Disease score & CI \\
\hline KAUZ/STAR/3/MUNIA/ALTAR84//MILAN/4/LEITH-1 & $15 \mathrm{MS}$ & 12 & 0 & 0 & $15 \mathrm{MR}$ & 6 & $10 \mathrm{MR}$ & 4 \\
\hline 22SAWSN142/3/PASTOR//MUNIA/ALTAR84/4/SHAMISS-3 & $10 \mathrm{MR}$ & 4 & $10 \mathrm{~S}$ & 10 & 0 & 0 & $10 \mathrm{MR}$ & 4 \\
\hline $\begin{array}{l}\text { PASTOR//HXL7573/2*BAU/3/SOKOLL/WBLL1/4/SAFI- } \\
\text { 1//NS732/HER/3/SAADA }\end{array}$ & $5 \mathrm{MR}$ & 2 & 0 & 0 & 0 & 0 & $15 \mathrm{MR}$ & 6 \\
\hline $\begin{array}{l}\text { PASTOR//HXL7573/2*BAU/3/SOKOLL/WBLL1/4/SAFI- } \\
\text { 1//NS732/HER/3/SAADA }\end{array}$ & $10 \mathrm{MS}$ & 8 & 0 & 0 & $15 \mathrm{MR}$ & 6 & $5 \mathrm{MR}$ & 2 \\
\hline $\begin{array}{l}\text { KIRITATI/4/SERI.1B*2/3/KAUZ*2/BOW//KAUZ/5/SHUHA- } \\
\text { 4/CHAM-8 }\end{array}$ & 0 & 0 & $10 \mathrm{MS}$ & 8 & 0 & 0 & 10MR & 4 \\
\hline $\begin{array}{l}\text { FARIS22/4/BOW/PRL//BUC/3/WH576/5/NINGMAI9558//CHIL/C } \\
\text { HUM18 }\end{array}$ & 10MR & 4 & 10MS & 8 & 20MR & 8 & 10MR & 4 \\
\hline P1.861/RDWG//ESWYT99\#18/ARRIHANE/3/PFAU/MILAN & $10 \mathrm{MS}$ & 8 & $5 \mathrm{MS}$ & 4 & 20MR & 8 & 40MS & 32 \\
\hline PFAU/MILAN//ABIER-2/3/SHUHA3//TURACO/CHIL & $10 \mathrm{MS}$ & 8 & $5 \mathrm{MS}$ & 4 & $5 \mathrm{MR}$ & 2 & $25 \mathrm{MR}$ & 10 \\
\hline OPATA/RAYON//KAUZ/3/ESWYT99\#18/ARRIHANE/4/SOSSI-3 & $10 \mathrm{~S}$ & 10 & $5 \mathrm{MR}$ & 2 & $10 \mathrm{MR}$ & 4 & $10 \mathrm{MR}$ & 4 \\
\hline HIDASSE & $10 \mathrm{MS}$ & 8 & $5 \mathrm{MS}$ & 4 & 10MR & 4 & 20MR & 8 \\
\hline OPATA/RAYON//KAUZ/3/ESWYT99\#18/ARRIHANE/4/SOSSI- & $25 \mathrm{~S}$ & 25 & 0 & 0 & 10MR & 4 & 10MR & 4 \\
\hline $\begin{array}{l}\text { FARIS22/4/BOW/PRL//BUC/3/WH576/5/NINGMAI9558//CHIL/C } \\
\text { HUM18 }\end{array}$ & $10 \mathrm{MS}$ & 8 & $5 \mathrm{MS}$ & 4 & $15 \mathrm{MR}$ & 6 & $5 \mathrm{MR}$ & 2 \\
\hline
\end{tabular}




\begin{tabular}{|c|c|c|c|c|c|c|c|c|}
\hline \multirow{2}{*}{ Pedigree } & \multicolumn{2}{|c|}{2016 (Stem Rust) } & \multicolumn{2}{|c|}{2017 (Stem Rust) } & \multicolumn{2}{|c|}{2016 (Yellow Rust) } & \multicolumn{2}{|c|}{2016 (Yellow Rust) } \\
\hline & Disease score & CI & Disease score & CI & Disease score & CI & Disease score & CI \\
\hline WBLL1 $* 2 /$ BRAMBLING//ZAFIR-3 & $20 \mathrm{MS}$ & 16 & $10 \mathrm{MS}$ & 8 & 10MR & 4 & $10 \mathrm{MR}$ & 4 \\
\hline WEAVER/TSC//WEAVER/3/WEAVER/4/WAXWING/5/DURRA-8 & $10 \mathrm{MS}$ & 8 & 0 & 0 & 0 & 0 & $10 \mathrm{MR}$ & 4 \\
\hline $\begin{array}{l}\text { REBWAH13/3/CMH81.38/2*KAUZ//ATTILA/4/URES/BOW//OPA } \\
\text { TA/3/HD2206/HORK' }\end{array}$ & $30 \mathrm{MS}$ & 24 & $10 \mathrm{MS}$ & 8 & 0 & 0 & $10 \mathrm{MR}$ & 4 \\
\hline WATAN-6/ETBW4919//ZAKIA-14 & $5 \mathrm{MR}$ & 2 & 0 & 0 & 0 & 0 & $5 \mathrm{MR}$ & 2 \\
\hline KAUZ/STAR//ETBW4920/3/QAMAR-2 & $5 \mathrm{MR}$ & 2 & 0 & 0 & 0 & 0 & $10 \mathrm{MR}$ & 4 \\
\hline ASEEL-1//MILAN/PASTOR/3/SHAMISS-3 & 0 & 0 & 0 & 0 & 0 & 0 & $10 \mathrm{MR}$ & 4 \\
\hline ZERBA-6/FLAG6/3/TAM200/PASTOR//TOBA97 & 0 & 0 & $5 \mathrm{MS}$ & 4 & 0 & 0 & $5 \mathrm{MR}$ & 2 \\
\hline KINGBIRD & $10 \mathrm{MS}$ & 8 & $5 \mathrm{MR}$ & 2 & $5 \mathrm{MR}$ & 2 & $15 \mathrm{MR}$ & 6 \\
\hline ZERBA-6/FLAG6/3/TAM200/PASTOR//TOBA97 & $5 \mathrm{MR}$ & 2 & $5 \mathrm{MS}$ & 4 & 0 & 0 & $5 \mathrm{MR}$ & 2 \\
\hline ZERBA-6/FLAG6/3/TAM200/PASTOR//TOBA97 & 0 & 0 & 0 & 0 & 0 & 0 & $5 \mathrm{MR}$ & 2 \\
\hline SUDAN\#3/SHUHA-6//FLAG-5/7/CHAM- & $10 \mathrm{MR}$ & 4 & 0 & 0 & $10 \mathrm{MR}$ & 4 & $10 \mathrm{MR}$ & 4 \\
\hline TEMPORALERAM $87 * 2 / \mathrm{KONK} / /$ VENAC & $15 \mathrm{MS}$ & 8 & $10 \mathrm{MS}$ & 8 & 0 & 0 & $5 \mathrm{MR}$ & 2 \\
\hline KINGBIRD/IZAZ-11 & $5 \mathrm{MS}$ & 4 & 0 & 0 & 0 & 0 & $10 \mathrm{MR}$ & 4 \\
\hline KINGBIRD/IZAZ-11 & $10 \mathrm{MS}$ & 8 & $5 \mathrm{MS}$ & 4 & $5 \mathrm{MR}$ & 2 & $10 \mathrm{MR}$ & 4 \\
\hline KINGBIRD/IZAZ-11 & $15 \mathrm{MS}$ & 12 & 0 & 0 & 0 & 0 & $10 \mathrm{MR}$ & 4 \\
\hline KINGBIRD/IZAZ-11 & $15 \mathrm{MS}$ & 12 & 0 & 0 & 0 & 0 & $10 \mathrm{MR}$ & 4 \\
\hline KINGBIRD/IZAZ-11 & $15 \mathrm{MS}$ & 12 & $5 \mathrm{MR}$ & 2 & $15 \mathrm{MR}$ & 6 & $15 \mathrm{MR}$ & 6 \\
\hline SHORIMA & 0 & 0 & 0 & 0 & $15 \mathrm{MR}$ & 6 & $15 \mathrm{MR}$ & 6 \\
\hline $\begin{array}{l}\text { MILAN/SHA7/3/THB/CEP7780//SHA4/LIRA/4/SHA4/CHIL/5/FA } \\
\text { RIS-6 }\end{array}$ & $10 \mathrm{MS}$ & 8 & 0 & 0 & $15 \mathrm{MR}$ & 6 & $10 \mathrm{MR}$ & 4 \\
\hline 22SAWSN-142/3/PASTOR//MUNIA/ALTAR84/4/SHAMISS-3 & $20 \mathrm{~S}$ & 20 & $5 \mathrm{MS}$ & 4 & 0 & 0 & $10 \mathrm{MR}$ & 4 \\
\hline 22SAWSN-142/3/PASTOR//MUNIA/ALTAR84/4/SHAMISS-3 & $10 \mathrm{MS}$ & 8 & 0 & 0 & $15 \mathrm{MR}$ & 6 & 30MS & 24 \\
\hline 22SAWSN-142/3/PASTOR//MUNIA/ALTAR84/4/SHAMISS-3 & $15 \mathrm{MS}$ & 12 & $5 \mathrm{MS}$ & 4 & $5 \mathrm{MR}$ & 2 & $10 \mathrm{MR}$ & 4 \\
\hline SKAUZ/2*STAR//ACHTAR/INRA & $15 \mathrm{MS}$ & 12 & $5 \mathrm{MR}$ & 2 & $15 \mathrm{MR}$ & 6 & $10 \mathrm{MR}$ & 4 \\
\hline $\begin{array}{l}\text { QT6581/4/PASTOR//SITE/MO/3/CHEN/AEGILOPSSQUARROS } \\
\text { A(TAUS)//BCN/5/PAVON76/JADIDA-2 }\end{array}$ & $10 \mathrm{MS}$ & 8 & 0 & 0 & $20 \mathrm{MR}$ & 8 & $10 \mathrm{MR}$ & 4 \\
\hline $\begin{array}{l}\text { QT6581/4/PASTOR//SITE/MO/3/CHEN/AEGILOPSSQUARROS } \\
\text { A(TAUS)//BCN/5/PAVON76/JADIDA-2 }\end{array}$ & $15 \mathrm{MR}$ & 6 & 0 & 0 & 0 & 0 & $10 \mathrm{MR}$ & 4 \\
\hline $\begin{array}{l}\text { SHARP/3/PRL/SARA//TSI/VEE\#5/5/VEE/LIRA//BOW/3/BCN/4/ } \\
\text { KAUZ/6/QAFZAH-4/3/VEE\#7//MT773/EMU'S' }\end{array}$ & $10 \mathrm{MR}$ & 4 & $5 \mathrm{MS}$ & 4 & 0 & 0 & $15 \mathrm{MR}$ & 6 \\
\hline PBW343/FLAG-4//QADANFER-4 & 0 & 0 & $5 \mathrm{MR}$ & 2 & $15 \mathrm{MR}$ & 6 & 20MR & 8 \\
\hline HIDASSE & $10 \mathrm{MR}$ & 4 & $5 \mathrm{MS}$ & 4 & $15 \mathrm{MR}$ & 6 & $15 \mathrm{MR}$ & 6 \\
\hline DEBEIRA/FLAG-6//SHUHA-1/DORG-1 & $10 \mathrm{~S}$ & 10 & 0 & 0 & $10 \mathrm{MR}$ & 4 & $10 \mathrm{MR}$ & 4 \\
\hline PFAU/MILAN//ABIER-2/3/SHUHA3//TURACO/CHIL & 0 & 0 & 0 & 0 & $5 \mathrm{MR}$ & 2 & 20MR & 8 \\
\hline RABIH-10/ETBW4922//KAUZ'S'/FLORKWA-1 & $5 \mathrm{MR}$ & 2 & 0 & 0 & $20 \mathrm{MS}$ & 16 & 40MR & 16 \\
\hline $\begin{array}{l}\text { THELIN/WAXWING//ATTILA*2/PASTOR/3/INQALAB91*2/TU } \\
\text { KURU9Y-0B }\end{array}$ & $10 \mathrm{MR}$ & 4 & $5 \mathrm{MS}$ & 4 & $15 \mathrm{MR}$ & 6 & $10 \mathrm{MR}$ & 4 \\
\hline $\begin{array}{l}\text { THELIN/WAXWING//ATTILA*2/PASTOR/3/INQALAB91*2/TU } \\
\text { KURU9Y-0B }\end{array}$ & $5 \mathrm{MR}$ & 2 & $5 \mathrm{MS}$ & 4 & $15 \mathrm{MR}$ & 6 & $10 \mathrm{MR}$ & 4 \\
\hline SUDAN\#3/SHUHA-6//FLAG5/3/PFAU/MILAN & 0 & 0 & 0 & 0 & $5 \mathrm{MR}$ & 2 & $5 \mathrm{MR}$ & 2 \\
\hline $\begin{array}{l}\text { THELIN/WAXWING//ATTILA*2/PASTOR/3/INQALAB91*2/TU } \\
\text { KURU9Y-0B }\end{array}$ & $10 \mathrm{MS}$ & 8 & $10 \mathrm{MR}$ & 4 & $10 \mathrm{MR}$ & 2 & $10 \mathrm{MR}$ & 4 \\
\hline $\begin{array}{l}\text { ATTILA/3*BCN//MILAN/DUCULA/7/BACANORA86/6/SN64/H } \\
\text { N4//REX/3/EDCH/MEX/4/SLS'S'/5/BOW'S' }\end{array}$ & $10 \mathrm{MS}$ & 12 & 0 & 0 & 0 & 0 & $10 \mathrm{MR}$ & 4 \\
\hline $\begin{array}{l}\text { HUBARA-1//ACHTAR/INRA1764/7/CHAM- } \\
\text { 8/6/SAKER'S'/5/RBS/ANZA/3/KVZ/HYS//YMH/TOB/4/BOW'S' }\end{array}$ & 0 & 0 & 0 & 0 & $15 \mathrm{MR}$ & 6 & $35 \mathrm{MR}$ & 14 \\
\hline KINGBIRD & $10 \mathrm{MS}$ & 8 & $15 \mathrm{MS}$ & 12 & $15 \mathrm{MR}$ & 6 & $10 \mathrm{MR}$ & 4 \\
\hline $\begin{array}{l}\text { HUBARA-1//ACHTAR/INRA1764/7/CHAM- } \\
\text { 8/6/SAKER'S'/5/RBS/ANZA/3/KVZ/HYS//YMH/TOB/4/BOW'S' }\end{array}$ & 0 & 0 & $15 \mathrm{MS}$ & 12 & $10 \mathrm{MR}$ & 4 & $25 \mathrm{MR}$ & 10 \\
\hline PFAU/MILAN//FUNGMAI24/3/ATTILA*2/CROW & $15 \mathrm{MS}$ & 12 & $5 \mathrm{MR}$ & 2 & 0 & 0 & $5 \mathrm{MR}$ & 2 \\
\hline WEAVER/TSC//WEAVER/3/WEAVER/4/WAXWING/5/DURRA-8 & $10 \mathrm{MR}$ & 4 & 0 & 0 & 20MR & 8 & $50 \mathrm{MS}$ & 40 \\
\hline WEAVER/TSC//WEAVER/3/WEAVER/4/WAXWING/5/DURRA-8 & $5 \mathrm{MR}$ & 2 & $10 \mathrm{MS}$ & 8 & 20MR & 8 & $50 \mathrm{MS}$ & 40 \\
\hline $\begin{array}{l}\text { REBWAH13/3/CMH81.38/2*KAUZ//ATTILA/4/URES/BOW//OPA } \\
\text { TA/3/HD2206/HORK' }\end{array}$ & $10 \mathrm{MS}$ & 8 & 0 & 0 & 20MR & 8 & $25 \mathrm{MR}$ & 10 \\
\hline KOUKAB-1/ETBW4920//PAVONF76 & $5 \mathrm{MR}$ & 2 & 0 & 0 & $15 \mathrm{MR}$ & 6 & 20MR & 8 \\
\hline CHAM-8/RUTH-3//ZAIN-2 & $15 \mathrm{MS}$ & 12 & $5 \mathrm{MR}$ & 2 & $10 \mathrm{MR}$ & 4 & $10 \mathrm{MR}$ & 4 \\
\hline ZERBA-6/FLAG6/3/TAM200/PASTOR//TOBA97 & 0 & 0 & 0 & 0 & 0 & 0 & $5 \mathrm{MR}$ & 2 \\
\hline $\begin{array}{l}\text { SERI.1B//KAUZ/HEVO/3/AMAD/4/ESWYT99\#18/ARRIHANE/5 } \\
\text { /SKAUZ/BAV92 }\end{array}$ & 0 & 0 & 0 & 0 & 0 & 0 & $5 \mathrm{MR}$ & 2 \\
\hline SHORIMA & 0 & 0 & $5 \mathrm{MS}$ & 4 & $10 \mathrm{MR}$ & 4 & $20 \mathrm{MR}$ & 8 \\
\hline MO88/MILAN//ETBW4922/3/(4)EALME4SA-464 & 0 & 0 & $5 \mathrm{MS}$ & 4 & 20MR & 8 & $25 \mathrm{MR}$ & 10 \\
\hline FARIS-17//PFAU/MILAN/3/SOSSI-3 & 0 & 0 & 0 & 0 & $15 \mathrm{MR}$ & 6 & $10 \mathrm{MR}$ & 4 \\
\hline TRAP\#1/BOW//PFAU/3/MILAN/4/ETBW & 0 & 0 & 0 & 0 & 0 & 0 & $5 \mathrm{MR}$ & 2 \\
\hline $\begin{array}{l}\text { SUDAN\#3/SHUHA-6//FLAG-5/7/CHAM- } \\
\text { 8/6/SAKER'S'/5/RBS/ANZA/3/KVZ/HYS//YMH/TOB/4/BOW'S' }\end{array}$ & 0 & 0 & $5 \mathrm{MS}$ & 4 & $10 \mathrm{MR}$ & 4 & $10 \mathrm{MR}$ & 4 \\
\hline
\end{tabular}




\begin{tabular}{|c|c|c|c|c|c|c|c|c|}
\hline \multirow{2}{*}{ Pedigree } & \multicolumn{2}{|c|}{2016 (Stem Rust) } & \multicolumn{2}{|c|}{2017 (Stem Rust) } & \multicolumn{2}{|c|}{2016 (Yellow Rust) } & \multicolumn{2}{|c|}{2016 (Yellow Rust) } \\
\hline & Disease score & CI & Disease score & CI & Disease score & CI & Disease score & CI \\
\hline KA/NAC//SERI/RAYON/3/GOUMRIA-14 & $5 \mathrm{MR}$ & 2 & $5 \mathrm{MR}$ & 2 & $15 \mathrm{MR}$ & 6 & $15 \mathrm{MR}$ & 6 \\
\hline $\begin{array}{l}\text { BABAX/LR42//BABAX*2/3/VIVITSI/4/SERI.1B*2/3/KAUZ*2/B } \\
\text { OW//KAUZ }\end{array}$ & 0 & 0 & 0 & 0 & 20MR & 8 & $10 \mathrm{MR}$ & 4 \\
\hline KINGBIRD/3/NESMA $* 2 / 14-2 / / 2 *$ SAFI-3 & $15 \mathrm{~S}$ & 15 & 0 & 0 & $20 \mathrm{MR}$ & 8 & $25 \mathrm{MR}$ & 10 \\
\hline P1.861/RDWG//ESWYT99\#18/ARRIHANE/3/PFAU/MILAN & $20 \mathrm{~S}$ & 20 & $10 \mathrm{MS}$ & 8 & 0 & 0 & $10 \mathrm{MR}$ & 4 \\
\hline P1.861/RDWG//ESWYT99\#18/ARRIHANE/3/PFAU/MILAN & 20MS & 16 & 0 & 0 & $15 \mathrm{MR}$ & 6 & $10 \mathrm{MR}$ & 4 \\
\hline HIDASSE & $10 \mathrm{MS}$ & 8 & $5 \mathrm{MR}$ & 2 & $10 \mathrm{MR}$ & 4 & $15 \mathrm{MR}$ & 6 \\
\hline FLORKWA- & & & & & & & & \\
\hline $\begin{array}{l}\text { 2/NJOROSD2/5/QT6581/4/PASTOR//SITE/MO/3/CHEN/AEGILO } \\
\text { PSSQUARROSA(TAUS)//BCN }\end{array}$ & 20MS & 8 & $20 \mathrm{MS}$ & 16 & $15 \mathrm{MR}$ & 6 & $5 \mathrm{MR}$ & 2 \\
\hline SUDAN\#3/SHUHA-6//FLAG5/3/PFAU/MILAN & $10 \mathrm{MS}$ & 8 & 0 & 0 & 0 & 0 & $5 \mathrm{MR}$ & 2 \\
\hline SUDAN\#3/SHUHA-6//FLAG-5/3/PFAU/MILAN & $5 \mathrm{MR}$ & 2 & 0 & 0 & 0 & 0 & $5 \mathrm{MR}$ & 2 \\
\hline ATTILA*2/AMAD//ENKOY/3/PFAU/MILAN & $5 \mathrm{MR}$ & 2 & $5 \mathrm{MS}$ & 4 & $5 \mathrm{MR}$ & 2 & $10 \mathrm{MR}$ & 4 \\
\hline ABU-REYAA-1/LEITH-1 & 30MS & 24 & 0 & 0 & $25 \mathrm{MR}$ & 10 & $35 \mathrm{MS}$ & 28 \\
\hline PFAU/MILAN//MOONTASIR-3 & $10 \mathrm{MR}$ & 4 & $5 \mathrm{MR}$ & 2 & 0 & 0 & $5 \mathrm{MR}$ & 2 \\
\hline CHAM-8/RUTH-3 & $25 \mathrm{MS}$ & 16 & $5 \mathrm{MR}$ & 2 & $10 \mathrm{MR}$ & 4 & $10 \mathrm{MR}$ & 4 \\
\hline $\begin{array}{l}\text { SERI.1B//KAUZ/HEVO/3/AMAD/4/ESWYT99\#18/ARRIHANE/5 } \\
\text { /SKAUZ/BAV92 }\end{array}$ & 20MS & 16 & 0 & 0 & $10 \mathrm{MR}$ & 4 & $10 \mathrm{MR}$ & 4 \\
\hline ZAIEM-8/KBG-01 & $15 \mathrm{MS}$ & 12 & $10 \mathrm{MS}$ & 8 & 0 & 0 & $10 \mathrm{MR}$ & 4 \\
\hline KINGBIRD & 20MS & 16 & $5 \mathrm{MS}$ & 4 & $15 \mathrm{MR}$ & 6 & $15 \mathrm{MR}$ & 6 \\
\hline $\begin{array}{l}\text { WBLL4//OAX93.24.35/WBLL1/4/SHUHA- } \\
\text { 1/3/MON'S'/ALD'S'//ALDAN'S'/IAS58 }\end{array}$ & 20MS & 16 & 0 & 0 & 20MR & 8 & $15 \mathrm{MR}$ & 6 \\
\hline $\begin{array}{l}\text { CHAM-10/3/PASTOR//MUNIA/ALTAR84/4/PFAU/MILAN } \\
\text { ZAFIR-3/TAZA- }\end{array}$ & $15 \mathrm{MS}$ & 12 & $5 \mathrm{MR}$ & 2 & $40 \mathrm{MS}$ & 32 & $30 \mathrm{MR}$ & 12 \\
\hline $\begin{array}{l}\text { 1/6/ND/VG9144//KAL/BB/3/YACO/4/CHIL/5/KAUZ*2//TC*6/RL } \\
\text { 5406(RL6043)/3/KAUZ }\end{array}$ & $15 \mathrm{MS}$ & 12 & $5 \mathrm{MS}$ & 4 & $30 \mathrm{MR}$ & 12 & $5 \mathrm{MR}$ & 2 \\
\hline ABU-REYAA-1/LEITH-1 & $15 \mathrm{MS}$ & 12 & 0 & 0 & 0 & 0 & $30 \mathrm{MR}$ & 12 \\
\hline $\begin{array}{l}\text { SERI.1B//KAUZ/HEVO/3/AMAD/4/ESWYT99\#18/ARRIHANE/5 } \\
\text { /SKAUZ/BAV92 }\end{array}$ & 20MS & 16 & $5 \mathrm{MS}$ & 4 & $5 \mathrm{MR}$ & 2 & $10 \mathrm{MR}$ & 4 \\
\hline OPATA/RAYON//KAUZ/3/ETBW4922/4/MI & $10 \mathrm{MR}$ & 4 & 0 & 0 & 0 & 0 & $20 \mathrm{MR}$ & 8 \\
\hline $\begin{array}{l}\text { PFAU/MILAN//FLAG-3/3/NEJMAH-9 } \\
\text { SHIHAB- }\end{array}$ & $10 \mathrm{MR}$ & 4 & $50 \mathrm{MS}$ & 40 & $15 \mathrm{MR}$ & 6 & $10 \mathrm{MR}$ & 4 \\
\hline $\begin{array}{l}\text { 19/KHIDER1/5/YANAC/3/PRL/SARA//TSI/VEE\#5/4/CROC- } \\
\text { 1/AE. SQUARROSA(224)//OPATA }\end{array}$ & 10MS & 8 & $5 \mathrm{MS}$ & 4 & $20 \mathrm{MR}$ & 8 & $10 \mathrm{MR}$ & 4 \\
\hline $\begin{array}{l}\text { HUITES/4/CS/TH. } \\
\text { SC//3*PVN/3/MIRLO/BUC/5/ETBW4922/6/QADANFER-4 }\end{array}$ & $10 \mathrm{MS}$ & 8 & $5 \mathrm{MR}$ & 2 & 20MSMR & 16 & 40MS & 32 \\
\hline SHORIMA & 0 & 0 & $5 \mathrm{MR}$ & 2 & $15 \mathrm{MR}$ & 6 & $15 \mathrm{MR}$ & 6 \\
\hline $\begin{array}{l}\text { HUITES/4/CS/TH. } \\
\text { SC//3*PVN/3/MIRLO/BUC/5/ETBW4922/6/QADANFER-4 }\end{array}$ & 0 & 0 & $5 \mathrm{MR}$ & 2 & 0 & 0 & $10 \mathrm{MR}$ & 4 \\
\hline FLORKWA-2/85Z1284//ETBW4920/3/LOULOU-18 & $15 \mathrm{MS}$ & 12 & 0 & 0 & 0 & 0 & $5 \mathrm{MR}$ & 2 \\
\hline $\begin{array}{l}\text { PASTOR//HXL7573/2*BAU/3/SOKOLL/WBLL1/4/SAFI- } \\
\text { 1//NS732/HER/3/SAADA }\end{array}$ & $10 \mathrm{MS}$ & 8 & 0 & 0 & 0 & 0 & $10 \mathrm{MR}$ & 4 \\
\hline $\begin{array}{l}\text { PASTOR//HXL7573/2*BAU/3/SOKOLL/WBLL1/4/SAFI- } \\
\text { 1//NS732/HER/3/SAADA }\end{array}$ & $5 \mathrm{MR}$ & 2 & 0 & 0 & 0 & 0 & $5 \mathrm{MR}$ & 2 \\
\hline $\begin{array}{l}\text { PASTOR//HXL7573/2*BAU/3/SOKOLL/WBLL1/4/SAFI- } \\
\text { 1//NS732/HER/3/SAADA }\end{array}$ & $10 \mathrm{MR}$ & 4 & $5 \mathrm{MS}$ & 4 & 0 & 0 & $10 \mathrm{MR}$ & 2 \\
\hline $\begin{array}{l}\mathrm{PASTOR} / / \mathrm{HXL} 7573 / 2 * \mathrm{BAU} / 3 / \mathrm{SOKOLL} / \mathrm{WBLL} 1 / 4 / \mathrm{SAFI}- \\
\text { 1//NS732/HER/3/SAADA }\end{array}$ & 0 & 0 & $5 \mathrm{MS}$ & 4 & $15 \mathrm{MR}$ & 6 & $10 \mathrm{MR}$ & 2 \\
\hline KRICHAUFF/2*PASTOR//SHUHA8/DUCU & 0 & 0 & $10 \mathrm{MS}$ & 8 & $15 \mathrm{MR}$ & 6 & $5 \mathrm{MR}$ & 2 \\
\hline WAXWING*2/KUKUNA//SHUHA-4/CHAM- & 0 & 0 & 0 & 0 & $15 \mathrm{MR}$ & 6 & $10 \mathrm{MR}$ & 4 \\
\hline TEVEE-1/STAR'S'//ETBW4920/3/TEPOCA+LR34/2*BORL95 & 0 & 0 & $5 \mathrm{MS}$ & 4 & $5 \mathrm{MR}$ & 2 & $15 \mathrm{MR}$ & 6 \\
\hline HIDASSE & $5 \mathrm{MR}$ & 2 & $5 \mathrm{MR}$ & 2 & $20 \mathrm{MR}$ & 8 & $15 \mathrm{MR}$ & 6 \\
\hline $\begin{array}{l}\text { 22SAWSN- } \\
\text { 142/ETBW4921/6/HPO/TAN//VEE/3/2*PGO/4/MILAN/ }\end{array}$ & $10 \mathrm{MR}$ & 4 & 0 & 0 & 0 & 0 & $15 \mathrm{MR}$ & 6 \\
\hline $\begin{array}{l}\text { THELIN/WAXWING//ATTILA*2/PASTOR/3/INQALAB91*2/TU } \\
\text { KURU9Y-0B }\end{array}$ & 0 & 0 & $5 \mathrm{MS}$ & 4 & $10 \mathrm{MR}$ & 4 & $15 \mathrm{MR}$ & 6 \\
\hline $\begin{array}{l}\text { HUBARA-1//ACHTAR/INRA1764/7/CHAM- } \\
\text { 8/6/SAKER'S'/5/RBS/ANZA/3/KVZ/HYS//YMH/TOB/4/BOW'S' }\end{array}$ & $5 \mathrm{MR}$ & 2 & 0 & 0 & 0 & 0 & $25 \mathrm{MR}$ & 10 \\
\hline NJOROSD-2/SHIHAB-12 & 0 & 0 & $5 \mathrm{MR}$ & 2 & 0 & 0 & $5 \mathrm{MR}$ & 2 \\
\hline NJOROSD-7/3/VEE/TSI/F134.71/CROW & 0 & 0 & $5 \mathrm{MR}$ & 2 & $15 \mathrm{MR}$ & 6 & 40MS & 32 \\
\hline $\begin{array}{l}\text { SERI.1B//KAUZ/HEVO/3/AMAD/4/ESWYT99\#18/ARRIHANE/5 } \\
\text { /SKAUZ/BAV92 }\end{array}$ & 0 & 0 & 0 & 0 & $15 \mathrm{MS}$ & 12 & $10 \mathrm{MR}$ & 4 \\
\hline MO88/MILAN//ETBW4922/3/(4)EALME4SA-464 & 0 & 0 & $10 \mathrm{~S}$ & 10 & $15 \mathrm{MR}$ & 6 & $10 \mathrm{MR}$ & 4 \\
\hline ICBW206971//SHUHA-4/CHAM8/3/SIRAJ- & 0 & 0 & $5 \mathrm{MR}$ & 2 & 0 & 0 & $5 \mathrm{MR}$ & 2 \\
\hline ALMAZ-26//ACHTAR/INRA1764/3/QAMAR-6 & 0 & 0 & $5 \mathrm{MR}$ & 2 & $15 \mathrm{MR}$ & 6 & $5 \mathrm{MR}$ & 2 \\
\hline KINGBIRD & $15 \mathrm{MS}$ & 8 & $5 \mathrm{MS}$ & 4 & $20 \mathrm{MR}$ & 8 & $15 \mathrm{MR}$ & 6 \\
\hline
\end{tabular}




\begin{tabular}{|c|c|c|c|c|c|c|c|c|}
\hline \multirow{2}{*}{ Pedigree } & \multicolumn{2}{|c|}{2016 (Stem Rust) } & \multicolumn{2}{|c|}{2017 (Stem Rust) } & \multicolumn{2}{|c|}{2016 (Yellow Rust) } & \multicolumn{2}{|c|}{2016 (Yellow Rust) } \\
\hline & Disease score & CI & Disease score & CI & Disease score & CI & Disease score & CI \\
\hline NJOROSD-7/3/VEE/TSI/F134.71/CROW & $5 \mathrm{MR}$ & 2 & $5 \mathrm{MS}$ & 4 & 0 & 0 & 5MR & 2 \\
\hline $\begin{array}{l}\text { BABAX/LR42//BABAX*2/3/VIVITSI/4/SERI.1B*2/3/KAUZ*2/B } \\
\text { OW//KAUZ }\end{array}$ & 0 & 0 & $10 \mathrm{MS}$ & 10 & $30 \mathrm{MR}$ & 12 & 40MS & 32 \\
\hline $\begin{array}{l}\text { NESMA } * 2 / 14-2 / / 2 * \text { SAFI- } \\
\text { 3/4/PASTOR//HXL7573/2*BAU/3/WBLL1 }\end{array}$ & 0 & 0 & 0 & 0 & $15 \mathrm{MR}$ & 6 & $15 \mathrm{MR}$ & 6 \\
\hline SUDAN\#3/SHUHA-6//FLAG-5/3/PFAU/MILAN & $10 \mathrm{MS}$ & 8 & 0 & 0 & $35 \mathrm{MR}$ & 14 & $5 \mathrm{MR}$ & 2 \\
\hline $\begin{array}{l}\text { MEX94.27.1.20/3/SOKOLL//ATTILA/3*BCN/4/NESMA*2/14- } \\
\text { 2//2*SAFI-3 }\end{array}$ & $10 \mathrm{MR}$ & 4 & $5 \mathrm{MS}$ & 4 & 0 & 0 & $15 \mathrm{MR}$ & 6 \\
\hline $\begin{array}{l}\text { SERI*3//RL6010/4*YR/3/PASTOR/4/BAV92/5/ETBW4921/6/SA } \\
\text { MIRA-9 }\end{array}$ & 0 & 0 & $10 \mathrm{MS}$ & 10 & $10 \mathrm{MR}$ & 4 & $5 \mathrm{MR}$ & 2 \\
\hline $\begin{array}{l}\text { QT6581/4/PASTOR//SITE/MO/3/CHEN/AEGILOPSSQUARROS } \\
\text { A(TAUS)//BCN/5/PAVON76/JADIDA-2 }\end{array}$ & $10 \mathrm{MS}$ & 8 & $5 \mathrm{MR}$ & 2 & $10 \mathrm{MR}$ & 4 & $20 \mathrm{MR}$ & 8 \\
\hline $\begin{array}{l}\text { QT6581/4/PASTOR//SITE/MO/3/CHEN/AEGILOPSSQUARROS } \\
\text { A(TAUS)//BCN/5/PAVON76/JADIDA-2 }\end{array}$ & $5 \mathrm{MR}$ & 2 & $5 \mathrm{MR}$ & 2 & $10 \mathrm{MR}$ & 4 & $25 \mathrm{MR}$ & 10 \\
\hline $\begin{array}{l}\text { MEX94.27.1.20/3/SOKOLL//ATTILA/3*BCN/4/NESMA*2/14- } \\
\text { 2//2*SAFI-3 }\end{array}$ & $5 \mathrm{MR}$ & 2 & $5 \mathrm{MS}$ & 4 & 0 & 0 & $10 \mathrm{MR}$ & 4 \\
\hline SHORIMA & 0 & 0 & $5 \mathrm{MR}$ & 2 & $20 \mathrm{MR}$ & 8 & $15 \mathrm{MR}$ & 6 \\
\hline $\begin{array}{l}\text { QT6581/4/PASTOR//SITE/MO/3/CHEN/AEGILOPSSQUARROS } \\
\text { A(TAUS)//BCN/5/PAVON76/JADIDA-2 }\end{array}$ & 0 & 0 & $5 \mathrm{MR}$ & 2 & $10 \mathrm{MR}$ & 4 & $15 \mathrm{MR}$ & 6 \\
\hline WAXWING*2/VIVITSI//SHUHA- & $15 \mathrm{MR}$ & 6 & $5 \mathrm{MS}$ & 4 & 0 & 0 & 40MS & 32 \\
\hline BABAX/LR42//BABAX*2/3/KUKUNA/4/AT & $10 \mathrm{MR}$ & 4 & $5 \mathrm{MR}$ & 2 & $10 \mathrm{MR}$ & 4 & $10 \mathrm{MR}$ & 4 \\
\hline BABAX/LR42//BABAX*2/3/KUKUNA/4/ATTILA*2/CRO & $10 \mathrm{MR}$ & 4 & $10 \mathrm{MS}$ & 8 & 10MR & 4 & $10 \mathrm{MR}$ & 4 \\
\hline $\begin{array}{l}\text { FARIS-17//PFAU/MILAN/3/SOSSI-3 } \\
\text { ZAFIR-3/TAZA- }\end{array}$ & 0 & 0 & 0 & 0 & $20 \mathrm{MR}$ & 8 & 40MS & 28 \\
\hline $\begin{array}{l}\text { 1/6/ND/VG9144//KAL/BB/3/YACO/4/CHIL/5/KAUZ*2//TC*6/RL } \\
5406(\mathrm{RL} 6043) / 3 / \mathrm{KAUZ}\end{array}$ & $15 \mathrm{MS}$ & 12 & $5 \mathrm{MS}$ & 4 & 0 & 0 & $5 \mathrm{MR}$ & 2 \\
\hline WBLL1//TEVEE/KAUZ/3/MILAN/SHA7//POTAM*3KS811261-5 & $15 \mathrm{MS}$ & 12 & $5 \mathrm{MR}$ & 2 & 0 & 0 & $10 \mathrm{MR}$ & 4 \\
\hline $\begin{array}{l}\text { SERI.1B//KAUZ/HEVO/3/AMAD/4/ESWYT99\#18/ARRIHANE/5 } \\
\text { /SITTA/BUCHIN//CHIL/BOMB }\end{array}$ & $10 \mathrm{MS}$ & 8 & $5 \mathrm{MS}$ & 4 & 10MR & 4 & $10 \mathrm{MR}$ & 4 \\
\hline FLORKWA-2/85Z1284//ETBW4920/3/LOULOU-18 & 0 & 0 & $5 \mathrm{MR}$ & 2 & 0 & 0 & 0 & 0 \\
\hline $\begin{array}{l}\text { SKAUZ/2*STAR/5/JUN//MAYA/MON/3/PGO/4/MILAN/6/TEMP } \\
\text { ORALERAM87*2/KONK }\end{array}$ & 0 & 0 & $5 \mathrm{MS}$ & 4 & $35 \mathrm{MR}$ & 14 & $50 \mathrm{MS}$ & 40 \\
\hline SOKOLL/WBLL1/4/SERI.1B//KAUZ/HEVO/ & $10 \mathrm{MS}$ & 8 & $5 \mathrm{MR}$ & 2 & $20 \mathrm{MS}$ & 16 & $30 \mathrm{MR}$ & 12 \\
\hline
\end{tabular}

\section{Discussion}

Though wheat is an important crop in Ethiopia, its production has been challenged by yellow and stem rust diseases causing up to $100 \%$ yield losses in some years in the main wheat growing belts of the country. To date, more than 80 bread wheat varieties of CIMMYT and ICARDA origin have been released in Ethiopia. However, currently only few varieties such as Kubsa, Kekeba, Medawelabu etc are grown with the application of fungicides. Development and deployment of resistant varieties is one of the key strategy to control rust diseases for the very fact that it is cheaper and friendly to the environment. However, because of the coevolution of the host and the pathogen, resistance of the varieties get broken shortly (on average 5years) after release leading to the boom and bust cycle to continue. Development of resistant varieties with major and minor gene combinations helps to extend the duration of the variety in its resistance form. To this end, elite spring bread wheat genotypes from ICARDA were evaluated at Kulumsa research center to identify genotypes resistance to both rusts. In the present study although the spreader rows were infected with the heavy stem and yellow rust disease pressure during the years 2016/17 and 2017/18, most of the genotypes remained resistant for both diseases. The genotypes that showed less severity level $(\leq 10 \%)$ for stem and yellow rust may contain major genes of stem rust such as SR2, SR24, SR25 and yellow rust such as Yr5, Yr10, Yr15, Yr18 with many other minor gene combinations. The currently identified resistant genotypes have been also evaluated at Merchouch station in Morocco and Terbol station of ICARDA in Lebanon against the Yr27 and the warrior races of yellow rust. These genotypes showed high level of resistance in both locations indicating that they do combine resistance for both races. The results revealed that lowest and highest AUDPC was attained on genotypes that showed lowest and highest disease severity, respectively. These results are in line with findings of [35]. Most of the studied genotypes showed that late infection and slow growth of the pathogen. Such disease resistance potential are best qualities of a slow rusting genotypes [42] and resulted low values of AUDPC [23].

The negative association between yellow and stem rust showed that high yellow rust disease severity tended to show low stem rust severities due to low photosynthetic area [32] under sever yellow rust infection. The negative correlation of stem and yellow rust with grain yield and thousand kernel weight revealed that the two rusts directly affects the grain quality leading to shriveling of wheat grains [28].

\section{Conclusion and Recommendation}

The current study has clearly indicated the presence of 
genetic variability for resistance to stem and yellow rusts within the elite genotypes of ICARDA origin. Among the many genotypes, we have identified ASEEL1//MILAN/PASTOR/3/SHAMISS3, ZERBA6/FLAG6/3/TAM200/PASTOR//TOBA97, ZERBA6/FLAG6/3/TAM200/PASTOR//TOBA97, NJOROSD-2/SHIHAB-12 and ICBW206971//SHUHA 4/CHAM8/3/SIRAJ as the top 5 genotypes with high level of resistance for both yellow rust and stem rust diseases. These genotypes shall be included in the national/regional variety trials for further evaluation to their adaptation and agronomic performance in the major wheat growing regions of Ethiopia for potential release. These genotypes are also recommended for parentage purposes in the wheat breeding programs at ICARDA and Kulumsa and other breeding programs in the region. Ethiopia is the hot spot for rust diseases and hence there is change of races frequently. It is therefore important to continue development and deployment of resistant varieties across different regions with application of fungicides to reduce the disease pressure. Pyramiding of major genes with minor genes through the application of molecular markers and key location phenotyping should be key strategies to continue in order to develop high yielding varieties with durable resistance to the major rusts.

\section{Acknowledgements}

The authors would like to thank ICARDA for financial support and Kulumsa Center for providing land to undertake this experiment.

\section{References}

[1] Beard, C., Jayasena, K., Thomas, G., and Loughman, R. 2006. Managing Stem Rust of Wheat. Plant Pathology, Department of Agriculture, Western Australia. Farmnote, 73.

[2] Tadesse, W., Bishaw, Z. andAssefa, S. (2019), "Wheat production and breeding in Sub Saharan Africa: Challenges and opportunities in the face of climate change", International Journal of Climate Change Strategies and Management, Vol. 11 No. 5, pp. 696-715. https://doi. org/10.1108/IJCCSM-022018-0015.

[3] Braun, H. J., Atlin, G., andPayne, T. 2010. Multi-location testing as a tool to identify plant response to global climate change. Climate Change and Crop Production page 115to138.

[4] ChenS., Zhang Rouse, W. Bolus., S., Rous, M. N., and Dubcovsky, J. (2018). Identification and characterization of wheat stem rust resistance gene $\mathrm{Sr} 21$ effective against the Ug99 race group at high temperature. PLoS Genet. 14: e 1007287. doi: 10. 1371 /journal. pgen. 1007287.

[5] Central Statistical Agency. 2017. The Federal Democratic Republic of Ethiopia, Central Statistical Agency, Agricultural Sample Survey 2016/17 (2009E. C.), VolumeI, Report on area and production of major crops (private peasant holdings, meher season). Statistical Bulletin 584, The Federal Democratic Republic of Ethiopia, Addis Ababa, Ethiopia.

[6] Chen XM. Epidemiology and control of stripe rust on wheat
(Pucciniastriiformisf. sp. Tritici) on wheat. Can J Plant Pathol. 2005; 27 (3): 314-337.

[7] Ever smeyer, M. G. \& Kramer, C. L. (2000). Epidemiology of wheat leaf and stem rust in the central great plains of USA.

[8] FAO. 2014. FAOSTAT data base. FAO, Rome. http: //faostat. fao. org / (accessed 8 July2014).

[9] Leonard, K. J. \&Szabo, L. J. Stem rust of small grains and grasses caused by Pucciniagraminis. Mol. Plant Pathol. 6, 99$111(2005)$.

[10] Lucas, H. 2012. The wheat initiative-an international research initiative for wheat improvement. Second Global Conference on Agricultural Research for Development (GCARD2). 29 October to 1 November, 2012, PuntadelEste, Uruguay.

[11] McIntosh, R. A., Wellings, C. R., and Park, R. F. 1995. Wheat Rusts: An Atlas of Resistance Genes. Plant Breeding Institute, The University of Sydney, CSIRO, Sydney, Australia.

[12] Negassa A, Shiferaw B, JawooK, Sonder K, Smale M, Braun HJ, Gbegbelegbe S, ZheGuo, Hodson D, Wood S, Payne T. and Abeyo B. 2013. The potential for wheat productionin Africa: Analysis of biophysical suitability and economic profitability. Centro International de Mejoramientode Maízy Trigo (CIMMYT), México.

[13] Pathan, A. K., and Park, R. F., (2006). Evaluation of seeding and adult plant resistant to leaf rust to European wheat cultivars Euphytica149, 327-342.

[14] Roelfs, A. P., R. P. Singh, and E. E. Saari. 1992. Rust Diseases of Wheat: Concepts and methods of disease management. Mexico, D. F: CIMMYT. 81pages.

[15] Singh R, Hodson P, Jin Y, Huerta-Espino J, Kinyua G, Wanyera R, Ward W (2006). Current status, likely migration and strategies to mitigate the threat to wheat production from race Ug99 (TTKS) of stem rust pathogen. CAB reviews: Perspectives in agriculture.

[16] Tesemma, T., and Mohammed, J. 1982. Review of wheat breeding in Ethiopia. Eth. J. Agric. Sci. 4: 11-24.

[17] Kebede, T., Geleta, B., Yai, B., and Badebo, A. 1995. Status of wheat rusts in the major producing regions of Ethiopia. Pages 180-184 in: Breeding for Disease Resistance with Emphasis on Durability. D. L. Danial, ed. Wageningen Agricultural University, Wageningen, The Netherlands.

[18] Kolmer J., Singh R., Garvin D., Viccars L., William H., Huerta-Espino J., et al. (2008). Analysis of the Lr34/Yr18 rust resistance region in wheat germplasm. Crop Sci. 48 18411852 10.2135/cropsci2007. 08. 0474.

[19] Esayas, A., 2003. Soils of Kulumsa agricultural research center. Technical Paper No. 76, The Federal Democratic Republic of Ethiopia, Ethiopian Agricultural Research Organization, July 2003, pp: 15.

[20] Peterson, R. F., A. B. Champbell, and A. E. Hannah. 1948. a diagrammatic scale for estimating rust intensity of leaves and stem of cereals. Can. J. Res. 26: 496-500. doi: 10. $1139 / \mathrm{cjr} 48 \mathrm{c}-03$.

[21] A. P. Roelfs, R. P. Singh, and E. E. Saari, Rust Diseases of Wheat: Concepts and Methods of Disease Management, CIMMYT, Mexico City, Mexico, 1992. 
[22] Olivera PD etal 2015. Phenotypic and genotypic characterization of race TKTTF of Pucciniagraminisf. sp. tritici that caused a wheat stem rust epidemic in southern Ethiopiain 2013/14Phytopathology105917-28.

[23] DrazIS, Abou- Elseoud MS, Kamara AM, Alaa-Eldein OA, El-bebany AF (2015). Screening of wheat genotypes for leaf rust resistance along with grain yield. Annals of Agricultural Sciences.

[24] Bekele H 2003 Short report on stripe rust and stem rust Proc. Agronomy Works hoped G Bedada (Addis Ababa, Ethiopia: Bale Agricultural Development Enterprise) pp6778BADE2003.

[25] SaariEE and Prescott JM 1985. World distribution in relation to economic losses. The Cereal Rusts. Vol2: Diseases, Distribution, Epidemiology and Controled AP Roelfs and WRB ushnell (Orlando: Academic) pp 259-98.

[26] Zadoks J C and Bouwman JJ1985. Epidemiology in Europe. The Cereal Rusts: Vol. II. Disease, Distribution, Epidemiology and Controled AP Roelfs and WRB ushnell (Orlando, FL: Academic) pp 329-69.

[27] Ali, S., Gladieux, P., Leconte, M., Gautier, A., Justesen, A. F., Hovmøller, M. S., Enjalbert, J., and deVallavieille-Pope, C. 2014. Origin, migration routes and worldwide population genetic structure of the wheat yellow rust pathogen Pucciniastriiformisf. sp. tritici. PLoS Pathog 10: 1-12.

[28] Atilaw, A., Bishaw, Z., Eticha, F., Gelalcha, S., Tadesse, Z., Aliye, S., Abdalla, O., Fikre, A., Ahmed, S., and Silim, S. 2014. Controlling wheat rusts and ensuring food security through deployment of resistant varieties in Ethiopia. Page19 in: Proc. $2^{\text {nd }}$ Int. Wheat Stripe Rust Symp. Izmir, Turkey.

[29] Abeyo, B., Hodson, D., Hundie, B., Woldeab, G., Girma, B., Badebo, A., Alemayehu, Y., Jobe, T., Tegegn, A., and Denbel, W. 2014. Cultivating success in Ethiopia.

[30] Bekele, H., Shambel, K., andDereje, H. 2002. Seasonal variations in the occurrence of wheat stripe rust in Bale highlands. Pest Manage. J. Ethiopia6: 65-72.

[31] Dereje, H., and Chemeda, F. 2006. Epidemics of striperust (Pucciniastriiformis) on common wheat (Triticumaestivum) in the highlands of Bale, southeastern Ethiopia. Crop Prot. 26: 1209-1218

[32] Bancal, M. O., Robert, C., and Ney, B., "Modelling wheat growth and yield losses from late epidemics of foliar diseases using loss of green area per layer and pre-anthesis reserves", Annals of Botany, 100. 777-789. 2007.
[33] Mulugeta, N. 1986. Estimates of phenotypic diversity and breeding potential of Ethiopian wheat. Hereditas 104: 41-48.

[34] AktasH, Zencirci N (2016). Stripe rust partial resistance increases spring bread wheat yield in South-Eastern Anatolia, Turkey. Journal of Phytopathology164: 1085-109.

[35] Chen, W., Wellings, C., Chen, X., Kang, Z., and Liu, T. 2014. Wheat stripe (yellow) rust caused by Pucciniastriiformisf. sp. tritici. Molecular Plant Pathology15: 433-446.

[36] Teklay A, Getaneh W, Woubit D (2012). Analysis of pathogen virulence of wheat stem rust and cultivar reaction to virulent races in Tigray, Ethiopia. African Journal of Plant Science 6: 244-250.

[37] Nazari K, Mafi M, Yahyaoui A, Singh RP, Park RF (2009). Detection of wheat stem rust (Pucciniagraminisf. sp. tritici) race TTKSK (Ug99) in Iran. Plant Disease 93: 317.

[38] Bekele E (1985). A review of research on diseases of barley, tef and wheat in Ethiopia. In: Tsedeke Abate (ed.), A review of crop protection research in Ethiopia. Institute of Agricultural Research (IAR), Ethiopia, pp79-107.

[39] Akfirat SF, AydinY, ErtugrulF, Hasancebi S, Budak H, Akan K, Mert Z, Bolat N, Uncuoglu AA. A microsatellite marker for yellow rust resistance in wheat. Cereal Res Commun. 2010; 38: 203-210. doi: 10. 1556/CRC. 38. 2010. 2.6

[40] R (RSoftware), 2016. R User's Guide: Statistics. R-3. 6. 0 Garrett Grolemund.

[41] Olivera, P., Szabo, L. J., Luster, D., and Jin, Y. 2017. Detection of virulent races from international populations of Pucciniagraminisf. sp. tritici. Phytopathology107: 12S.

[42] Herrera-Foessel, S. A., Lagudah, E. S., Huerta Espino, J., Hayden, M. J., Bariana, H. S., Singh, D., and Singh, R. P. 2010. New slow- rusting leaf rust and stripe rust resistance genes Lr67 and Yr46 in wheat are closely linked. Theor. Appl. Genet. 122: 239-249.

[43] Chen, X. M. 2005. Epidemiology and control of stripe rust [Pucciniastriiformisf. sp. tritici] on wheat. Canadian Journal of Plant Pathology 27: 314-337.

[44] Beddow, J. M., Pardey, P. G., Chai, Y., Hurley, T. M., Kriticos, D. J., Braun, H. J., Park, R. F., Cuddy, W. S. and Yonow, T. (2015). Research investment implications of shifts in the global geography of wheat Stripe rust. Nat. Plants, 1, 15132. 